\title{
Imaging Molecular Motion: Femtosecond X-Ray Scattering of an Electrocyclic Chemical Reaction
}

\author{
M. P. Minitti, ${ }^{1,}$ J. M. Budarz, ${ }^{1,2}$ A. Kirrander, ${ }^{3}$ J. S. Robinson, ${ }^{1}$ D. Ratner, ${ }^{1}$ T. J. Lane, ${ }^{1,4}$ D. Zhu, ${ }^{1}$ \\ J. M. Glownia, ${ }^{1}$ M. Kozina, ${ }^{1}$ H. T. Lemke, ${ }^{1}$ M. Sikorski, ${ }^{1}$ Y. Feng, ${ }^{1}$ S. Nelson, ${ }^{1}$ K. Saita, ${ }^{3}$ B. Stankus, ${ }^{2}$ \\ T. Northey, ${ }^{3}$ J. B. Hastings, ${ }^{1, \dagger}$ and P. M. Weber ${ }^{2, \$}$ \\ ${ }^{1}$ SLAC National Accelerator Laboratory, Menlo Park, California 94025, USA \\ ${ }^{2}$ Brown University, Department of Chemistry, Providence, Rhode Island 02912, USA \\ ${ }^{3}$ School of Chemistry, University of Edinburgh, Edinburgh EH9 3FJ, United Kingdom \\ ${ }^{4}$ Stanford University, Department of Chemistry, Stanford, California 94305, USA
}

(Received 28 February 2015; published 22 June 2015)

\begin{abstract}
Structural rearrangements within single molecules occur on ultrafast time scales. Many aspects of molecular dynamics, such as the energy flow through excited states, have been studied using spectroscopic techniques, yet the goal to watch molecules evolve their geometrical structure in real time remains challenging. By mapping nuclear motions using femtosecond x-ray pulses, we have created real-space representations of the evolving dynamics during a well-known chemical reaction and show a series of timesorted structural snapshots produced by ultrafast time-resolved hard x-ray scattering. A computational analysis optimally matches the series of scattering patterns produced by the $\mathrm{x}$ rays to a multitude of potential reaction paths. In so doing, we have made a critical step toward the goal of viewing chemical reactions on femtosecond time scales, opening a new direction in studies of ultrafast chemical reactions in the gas phase.
\end{abstract}

DOI: 10.1103/PhysRevLett.114.255501

PACS numbers: 61.05.cf, 33.15.-e, 41.60.Cr

For more than a century, chemists have explored chemical reactions that transform molecules from one structure to another. While mapping structure or function has become routine in many instances, understanding the chemical dynamics that connect structure and function remains, however, remarkably challenging [1]. A growing number of experiments are emerging that aim to map chemical reactions with spatial and temporal resolution, including molecular frame photoelectron spectroscopy [2], Coulomb explosion imaging [3-5], and ultrafast electron diffraction [6]. It is notable that the most important and frequently used techniques for the determination of static molecular structures, namely $\mathrm{x}$-ray scattering and nuclear magnetic resonance, are absent from this list.

$\mathrm{X}$-ray scattering is one of the most powerful techniques for structure determination, but the insufficient photon flux and too-long pulse durations have rendered time-dependent studies largely unfeasible. Some success has been attained using chopped $\mathrm{x}$-ray pulses from synchrotrons in the condensed phases for comparatively slow $(t \geq 50 \mathrm{ps})$ chemical processes $[7,8]$. Chemical reaction dynamics studies aimed at determining unique structural conformations benefit from dilute gases, where the reactions unfold uninhibited by perturbations from neighboring molecules or collisions. Unfortunately, the numbers of molecules in the interaction region in many gas-phase samples are often too low to provide sufficient signal with conventional x-ray sources, which also lack the time-resolution required for reactions that unfold on the femtosecond time scale. Impressive advances have been achieved using electron scattering [9-12].
Electron scattering has a qualitatively larger cross section than scattering of $\mathrm{x}$ rays, but space-charge interactions between electrons within a pulse make it difficult to reach the pulse durations needed to follow molecular motions.

This Letter demonstrates that x-ray scattering can be used as a tool to study gas-phase ultrafast chemical reactions. The realization of hard $\mathrm{x}$-ray free electron lasers (XFELs), notably the Linac Coherent Light Source (LCLS) in 2009, has made available sources with unprecedented $\mathrm{x}$-ray brightness. Their pulse durations are as short as a few femtoseconds and the intensity of a single x-ray pulse at LCLS is comparable to that available from synchrotron sources integrated over $1 \mathrm{~s}$. These parameters open up the possibility to apply ultrafast $\mathrm{x}$-ray sources to the study of chemical reaction dynamics in dilute gases $[13,14]$ and have now made it possible to observe molecular scattering patterns with a time resolution approaching the ultrashort duration of laser pulses [15]. Scattering experiments yield unique patterns that represent Fourier transforms of the molecular structure. Because scattering experiments directly probe molecular geometry, they are ideally suited for mechanistic studies on ultrafast time scales. We report here the observation of time-evolving x-ray scattering patterns from dilute gas phase molecules during a chemical reaction: the ultrafast ring-opening reaction of 1,3-cyclohexadiene (CHD) to form linear 1,3,5-hexatriene (HT).

The ring-opening reaction of CHD has intrigued scientists for many years. As a prototypical example of an electrocyclic reaction, it has played an important role in the understanding of a large class of organic reactions [16]. The reaction 
motif also features prominently in synthetic processes, photochemical switches, and natural product synthesis [17]. Upon excitation by an UV photon, the molecule slides down the potential energy surface of the $1 B$ electronic state and goes through a conical intersection to reach a steeply repulsive $2 A$ surface, from where it transitions via an avoided crossing to the ground state of the reaction product [17]. From spectroscopic experiments, the time scales are known [18-20] but the correlation of time constants to molecular structures has remained absent. By applying x-ray scattering, we are able to assign molecular structures to each time point of the reaction before the molecules reach a thermal equilibrium of conformational structures [21].

The experiment was performed at the X-ray PumpProbe (XPP) Instrument of LCLS [22]. Briefly, CHD vapor at a pressure of 3-4 torr, corresponding to only $\sim 1 \times 10^{17}$ molecules $/ \mathrm{cm}^{3}$, for a total of $\sim 8 \times 10^{12}$ total molecules in the interaction region, were introduced into a custom scattering chamber (see Fig. 1). The ring-opening reaction was initiated by the absorption of a $267 \mathrm{~nm}$ optical pump photon (65 fs, 4-8 $\mu \mathrm{J}, 100 \mu \mathrm{m}$ FWHM focus). The structural evolution of CHD to HT was observed by a timedelayed $\mathrm{x}$-ray probe $\left(8.3 \mathrm{keV}, 30 \mathrm{fs}, 10^{12}\right.$ photons/pulse, $30 \times 30 \mu \mathrm{m}$ FWHM focus) propagating collinearly with the pump laser. Scattering patterns were collected on a 2.3megapixel Cornell-SLAC Pixel Array Detector (CSPAD) imaging detector [23] as a function of time delay. For each pump-probe pair, precise relative arrival times of the $\mathrm{x}$-ray and optical pulses at the sample were monitored by a spectrally encoded cross correlator [24,25]. Fast data collection enabled the adjustment of experimental parameters, such as delay time and beam overlap, to optimize experimental conditions during the experimental runs.

The percentage changes in the measured scattering signal for momentum transfers between 1.0 and $4.2 \AA^{-1}$ as a function of delay between optical laser excitation and the x-ray probe are shown in Fig. 2. The changes are on the order of $1 \%$, which we estimate corresponds to an average of $7 \%$ of the molecules excited by the pump pulse. In the experiment, the percentage of molecules excited was deliberately kept low to minimize multiphoton processes. Immediately after optical excitation, the difference scattering signal appears as either positive or negative changes as a function of momentum transfer and time delay. No further changes were observed after approximately 200 fs. This is consistent with optical pump-probe experiments that have suggested that the molecule evolves on the excited electronic state surfaces for about 140 fs [14].

The time dependence of the scattering signal for specific momentum transfers is shown in Fig. 3(a). The analysis [Figs. 3(b) and 3(c)] shows that certain momentum transfers are associated with transient features as short-lived as

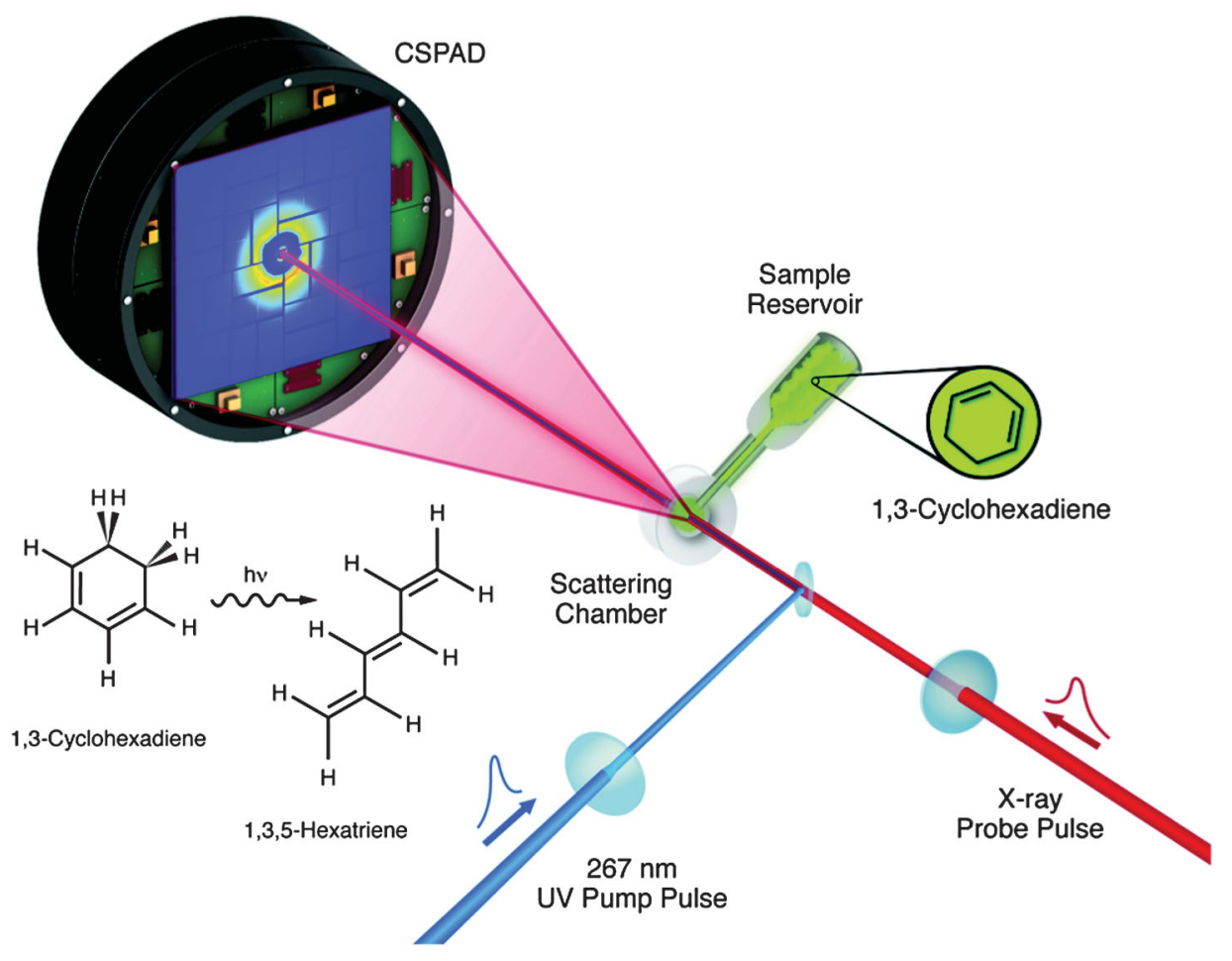

FIG. 1 (color). Experimental setup for ultrafast time resolved x-ray scattering studies. Low pressure 1,3-cyclohexadiene vapor (green) is introduced into the scattering cell via a needle valve from a room temperature sample reservoir. CHD is excited to the photoactive state by the UV optical pump pulse (blue), inducing the chemical reaction that leads to several conformers of 1,3,5-hexatriene. The reacting molecules are probed by diffracting photons from an $8.3 \mathrm{keV}$ x-ray pulse (red) that arrives with variable time delay relative the pump pulse, onto a large area pixel array detector. The time delay between the optical and x-ray pulses are varied to obtain the complete molecular movie. 


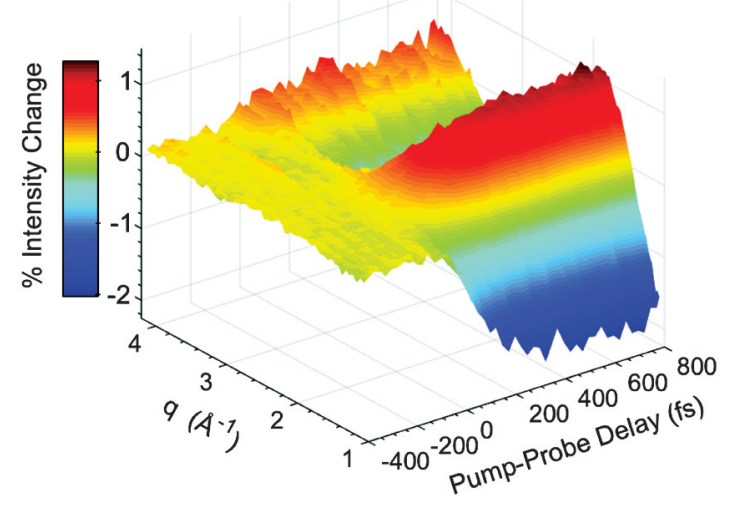

FIG. 2 (color). The time-dependent pump-probe signal, defined as the percent difference. The scattering patterns for positive delay times versus negative delay times [100*(laser on-laser off)/laser off], as a function of pump-probe delay time. The scattering signals change on the order of $1 \%$ to $2 \%$ as indicated by the color bar.

75 fs, while others have considerably longer lifetimes. The difference in the observed rates and lifetimes for various regions of momentum transfer [Figs. 3(b) and 3(c)] are simply attributed to the distances between non-neighboring carbons within CHD and HT, moving with respect to one another on different time scales. The shortest lived transients [Fig. 3(b)] exhibit lifetimes on the order of the dissociation reaction [14], suggesting that the experiment captures the CHD molecule as it reacts in the time domain.

The x-ray scattering results show that the structural part of the transformation of 1,3-cyclohexadiene to 1,3,5-hexatriene proceeds with an 80 fs time constant. This compares with the decay (140 fs) of the last electronic state populated in the reaction sequence and the point from where hexatriene is generated (the $2 A$ surface), as reported from spectroscopic experiments [17]. The time scale also compares with a $30 \mathrm{fs}$ lifetime of the initially excited $1 B$ electronic state, and the separately observed spectroscopic appearance time of structurally disperse hexatriene of $140 \mathrm{fs}$ [26]. The x-ray scattering experiment presented here, solely limited by the achievable momentum vector resolution, not only provides reliable time constants for the structural evolution of the reaction, but also allows one to calculate representative molecular structures along the reaction path consistent with the experiment.

To determine the time evolution of molecular structure implied by the experimental data, we start by calculating 100 trajectories, which represent a wide range of plausible reaction paths. Each trajectory is obtained with slightly different starting conditions sampled from a vibrational Wigner distribution of the $v=0$ vibrational state of the $S_{0}$ electronic ground state [27]. Trajectories are propagated using the multiconfigurational Ehrenfest method [28] with potential energies and nonadiabatic couplings obtained on-the-fly from SA3-CAS(6,4)-SCF/cc-pVDZ ab initio electronic structure calculations using MOLPRO [29]. The all-atom simulations do not assume any reduced representation or pre-existing reaction coordinate. Three electronic states are included: the ground state, the optically accessed $1 B$ state, and the $2 A$ state that is implicated in the Woodward-Hoffman mechanism of the electrocyclic reaction [16]. The rotationally averaged coherent scattering signal is obtained via the independent-atom model by [30]

$$
I(t, q)=\sum_{i=1}^{N_{\text {atom }}}\left|f_{i}^{0}(q)\right|+\sum_{i \neq j}^{N_{\text {atom }}} f_{i}^{0}(q) f_{j}^{0}(q) \frac{\sin q R_{i j}(t)}{q R_{i j}(t)},
$$

FIG. 3 (color). Time-dependent scattered signal intensities. (a) Line traces of the scattering signals at 2.1-2.5 $\AA$ (red squares) and 2.9-3.1 $\AA$ (blue circles), and fits (red and blue lines, respectively). (b), (c) The derivatives of the fits show transients of $82 \pm 24$ and $75 \pm 35 \mathrm{fs}$, respectively. Error bars for the fits in (a) and the uncertainties in (b),(c) are reported to $3 \sigma$, calculated from the frame-to-frame shot noise from the CSPAD detector. 
using published elastic scattering atomic form factors [31], $f_{i}^{0}(q)$, and internuclear distances, $R_{i j}(t)$, taken from our trajectories. Each trajectory constitutes a molecular reaction path with a complete but distinct set of interatomic distances. For visualization, it is helpful to represent the trajectories in terms of a single distance, that between the terminal carbons $\mathrm{C} 1$ and $\mathrm{C} 6$ in HT, which corresponds to the bond that breaks in CHD during the reaction. While all trajectories start near the $1.54 \AA$ bond distance of CHD [17], the trajectories diverge within the first 50 fs of the reaction, as can be seen in the Supplemental Material's Fig. 1 [32]. Some of the molecules remain initially in bonded form, but open up after one or more oscillations.

To determine the combination of trajectories that best describes the chemical reaction, we compare the experimental scattering patterns with scattering patterns calculated from the computed trajectories. Using a multistart nonlinear least-square optimization routine with a finitedifference gradient [47], we determined the weights on the trajectories which result in a signal that best matches the experimental data in Fig. 2. The optimization converges on a small number of trajectories (highlighted in the Supplemental Material's Fig. 2 [32]) with the four dominant trajectories having a combined weight of approximately $80 \%$, and the remaining $20 \%$ made up by four more trajectories. Based on the representation of x-ray scattering embodied by Eq. (1), these trajectories suffice to represent the experimentally observed data, within the approximations inherent in Eq. (1) and a classical representation of the chemical reaction. A direct comparison of the theoretical signal and the experimental data at particular time points is shown in Fig. 4. Movies showing the time-evolving molecular structures of the four dominant trajectories (bold lines in the Supplemental Material's Fig. 2) are available as extended data (see movies 1-4 [32]).

Our analysis shows that, upon absorption of the optical photon, the chemical reaction of 1,3-cyclohexadiene to 1,3,5-hexatriene starts with a rapid expansion of the carbon bonds of the cyclohexadiene ring. This expansion is related to the weakening of the chemical bonds due to the electronic excitation of the molecular $\pi$ bonds. Within one or two oscillations of the carbon skeleton, the C1-C6 chemical bond breaks as the terminal carbon atoms move perpendicular to the molecular plane along the reaction coordinate. It is already at this point that the terminal hydrogen atoms of the nascent hexatriene molecule align to conform to the Woodward-Hoffman rules [16]. Consequently, the stereochemical fate of the chemical reaction is sealed as early as $30 \mathrm{fs}$ after the optical excitation.

In summary, we report an ultrafast time-resolved gasphase $\mathrm{x}$-ray scattering experiment, recording the ringopening reaction of 1,3-cyclohexadiene using femtosecond hard x-ray pulses from LCLS, and thereby opening a new direction for time-resolved $\mathrm{x}$-ray scattering experiments for chemical dynamics. Time-dependent $\mathrm{x}$-ray scattering patterns from molecular structures as they evolve during

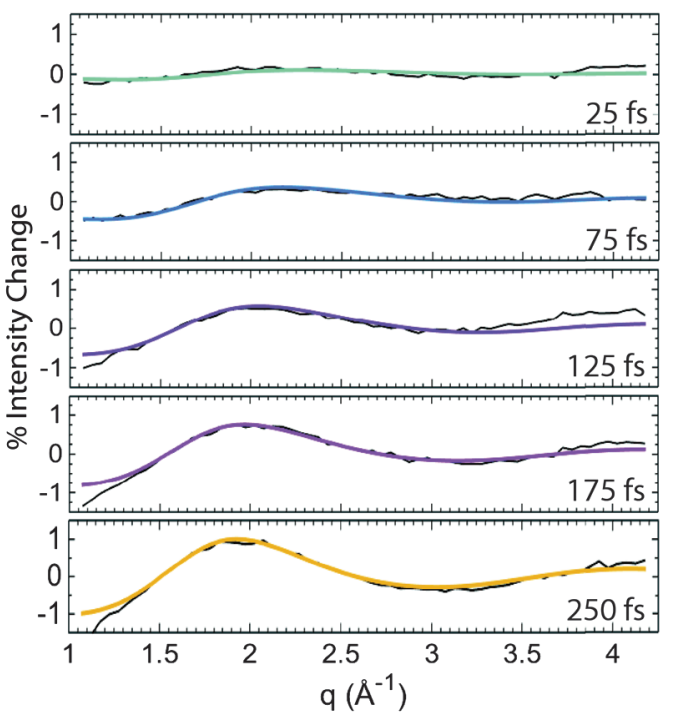

FIG. 4 (color). Experimental (black lines) and computational (thick colored lines) scattering signals for the first $250 \mathrm{fs}$ of the ring-opening reaction of 1,3-cylohexadiene.

chemical reactions can provide important feedback to mechanistic studies, as well as to computational methods that aim to elucidate chemical reaction mechanisms. Such data should prove quite useful to spectroscopic experiments, which associate specific spectral features with time points in the reaction. With the time-evolution of molecular structure available from $x$-ray scattering experiments, the spectroscopic results can be better matched to computer simulations, providing synergy between different experimental techniques and theory. In particular, important spectroscopic experiments that explore the path of the molecule through the conical intersections can be coupled with structural information. The time-dependent molecular structures during the electrocyclic ring-opening reaction of 1,3-cyclohexadiene to 1,3,5-hexatriene obtained in the present work will help form an important foundation for a significant body of future XFEL experimental and computational studies. We anticipate an exciting period of rapid developments in ultrafast $\mathrm{x}$-ray scattering experiments that push both temporal and spatial resolution boundaries, as well as in the theoretical and computational framework required to interpret these new experiments.

Portions of this research were carried out on the XPP Instrument at the Linac Coherent Light Source (LCLS), a division of SLAC National Accelerator Laboratory and an Office of Science user facility operated by Stanford University. P. M. W. acknowledges partial support by the National Science Foundation, Grant No. CBET1336105. A. K., K.S., and T.N. acknowledge Grants No. FP7-PEOPLE-2013-CIG-NEWLIGHT (European Union) and No. RPG-2013-365 (Leverhulme Trust), and helpful discussions with D. Shalashilin (Leeds).

M. P. M. and J. M. B. contributed equally to this work. 
*Corresponding author.

minitti@slac.stanford.edu

${ }^{\dagger}$ Corresponding author.

jbh@slac.stanford.edu

Corresponding author.

Peter_Weber@brown.edu

[1] A. H. Zewail, J. Phys. Chem. A 104, 5660 (2000).

[2] P. Hockett, C. Z. Bisgaard, O. J. Clarkin, and A. Stolow, Nat. Phys. 7, 612 (2011).

[3] H. Ibrahim, B. Wales, S. Beaulieu, B. E. Schmidt, N. Thiré, E. P. Fowe, É. Bisson, C. T. Hebeisen, V. Wanie, M. Giguére, J.-C. Kieffer, M. Spanner, A. D. Bandrauk, J. Sanderson, M. S. Schuurman, and F. Légaré, Nat. Commun. 5, 4422 (2014).

[4] J. L. Hansen, J. H. Nielsen, C. B. Madsen, A. T. Lindhardt, M. P. Johansson, T. Skrydstrup, L. B. Madsen, and H. Stapelfeldt, J. Chem. Phys. 136, 204310 (2012).

[5] L. Christensen, J. H. Nielsen, C. B. Brandt, C. B. Madsen, L. B. Madsen, C. S. Slater, A. Lauer, M. Brouard, M. P. Johansson, B. Shepperson, and H. Stapelfeldt, Phys. Rev. Lett. 113, 073005 (2014).

[6] C. J. Hensley, J. Yang, and M. Centurion, Phys. Rev. Lett. 109, 133202 (2012).

[7] H. Ihee, M. Lorenc, T. K. Kim, Q. Y. Kong, M. Cammarata, J. H. Lee, S. Bratos, and M. Wulff, Science 309, 1223 (2005).

[8] R. Neutze, R. Wouts, S. Techert, J. Davidsson, M. Kocsis, A. Kirrander, F. Schotte, and M. Wulff, Phys. Rev. Lett. 87, 195508 (2001).

[9] R. C. Dudek and P. M. Weber, J. Phys. Chem. A 105, 4167 (2001).

[10] J. B. Hastings, F. M. Rudakov, D. H. Dowell, J. F. Schmerge, J. D. Cardoza, J. M. Castro, S. M. Gierman, H. Loos, and P. M. Weber, Appl. Phys. Lett. 89, 184109 (2006).

[11] H. Ihee, V. A. Lobastov, U. M. Gomez, B. M. Goodson, R. Srinivasan, C. Y. Ruan, and A. H. Zewail, Science 291, 458 (2001).

[12] B. J. Siwick, J. R. Dwyer, R. E. Jordan, and R. J. D. Miller, Science 302, 1382 (2003).

[13] J. Küpper et al., Phys. Rev. Lett. 112, 083002 (2014).

[14] V. S. Petrović et al., Phys. Rev. Lett. 108, 253006 (2012).

[15] P. Emma et al., Nat. Photonics 4, 641 (2010).

[16] R. B. Woodward and R. Hoffmann, Angew. Chem., Int. Ed. Engl. 8, 781 (1969).

[17] S. Deb and P. M. Weber, Annu. Rev. Phys. Chem. 62, 19 (2011).

[18] M. Garavelli, C. S. Page, P. Celani, M. Olivucci, W. E. Schmid, S. A. Trushin, and W. Fuss, J. Phys. Chem. A 105, 4458 (2001).

[19] N. Kuthirummal, F. M. Rudakov, C. L. Evans, and P. M. Weber, J. Chem. Phys. 125, 133307 (2006).

[20] K. Kosma, S. A. Trushin, W. Fuss, and W. E. Schmid, Phys. Chem. Chem. Phys. 11, 172 (2009).

[21] C. Y. Ruan, V. A. Lobastov, R. Srinivasan, B. M. Goodson, H. Ihee, and A. H. Zewail, Proc. Natl. Acad. Sci. U.S.A. 98, 7117 (2001).

[22] S. Moeller et al., Nucl. Instrum. Methods Phys. Res., Sect. A 635, S6 (2011).

[23] H. T. Philipp, M. Hromalik, M. Tate, L. Koerner, and S. M. Gruner, Nucl. Instrum. Methods Phys. Res., Sect. A 649, 67 (2011).
[24] M. R. Bionta, H. T. Lemke, J. P. Cryan, J. M. Glownia, C. Bostedt, M. Cammarata, J.-C. Castagna, Y. Ding, D. M. Fritz, A. R. Fry, J. Krzywinski, M. Messerschmidt, S. Schorb, M. L. Swiggers, and R. N. Coffee, Opt. Express 19, 21855 (2011).

[25] M. Harmand, R. Coffee, M. R. Bionta, M. Chollet, D. French, D. Zhu, D. M. Fritz, H. T. Lemke, N. Medvedev, B. Ziaja, S. Toleikis, and M. Cammarata, Nat. Photonics 7, 215 (2013).

[26] C. C. Pemberton, Y. Zhang, K. Saita, A. Kirrander, and P. M. Weber, (to be published).

[27] R. C. Brown and E. J. Heller, J. Chem. Phys. 75, 186 (1981).

[28] K. Saita and D. V. Shalashilin, J. Chem. Phys. 137, 22 A506 (2012).

[29] Computer code MOLPRO, version 2012.1, a package of $a b$ initio programs, 2012.

[30] P. Debye, Ann. Phys. (Berlin) 351, 809 (1915).

[31] A. J.C. Wilson and E. Prince, International Tables for Crystallography: Volume C-Mathematical, Physical and Chemical Tables (Kluwer Academic Publishers, Dordrecht, 1999), Vol. C, p. 992.

[32] See Supplemental Material http://link.aps.org/supplemental/ 10.1103/PhysRevLett.114.255501, which includes Refs. [33-46], for details on experimental protocol, experimental data handling, and theoretical methods used to generate the data presented in the Letter.

[33] S. Herrmann, P. Hart, A. Dragone, D. Freytag, R. Herbst, J. Pines, M. Weaver, G. A. Carini, J. B. Thayer, O. Shawn, C. J. Kenney, and G. Haller, J. Phys. Conf. Ser. 493, 012013 (2014).

[34] U. Lorenz, K. B. Møller, and N. E. Henriksen, New J. Phys. 12, 113022 (2010).

[35] R. Srinivasan, V.A. Lobastov, C.-Y. Ruan, and A. H. Zewail, Helv. Chim. Acta 86, 1761 (2003).

[36] D. V. Shalashilin, J. Chem. Phys. 132, 244111 (2010).

[37] H. Tao, T. J. Martinez, H. Andersen, and P. H. Bucksbaum, First Principles Molecular Dynamics and Control of Photochemical Reactions (Stanford University, Stanford, CA, 2011).

[38] D. V. Shalashilin, Faraday Discuss. 153, 105 (2011).

[39] T. Northey, N. Zotev, and A. Kirrander, J. Chem. Theory Comput. 10, 4911 (2014).

[40] U. Lorenz, K. B. Møller, and N. E. Henriksen, Phys. Rev. A 81, 023422 (2010).

[41] N. E. Henriksen and K. B. Møller, J. Phys. Chem. B 112, 558 (2008).

[42] K. Møller and N. Henriksen, in Mol. Electron. Struct. Transit. Met. Complexes I, edited by D. M. P. Mingos, P. Day, and J. P. Dahl, Structure and Bonding 142 (Springer, Berlin, Heidelberg, 2012), pp. 185-211.

[43] G. Dixit, O. Vendrell, and R. Santra, Proc. Natl. Acad. Sci. U.S.A. 109, 11636 (2012).

[44] G. Dixit and R. Santra, J. Chem. Phys. 138, 134311 (2013).

[45] Computer code Matlab, 2012.

[46] S. Adachi, M. Sato, and T. Suzuki, J. Phys. Chem. Lett. 6, 343 (2015).

[47] M. P. Minitti, J. M. Budarz, A. Kirrander, J. Robinson, T. J. Lane, D. Ratner, K. Saita, T. Northey, B. Stankus, V. Cofer-Shabica, J. Hastings, and P. M. Weber, Faraday Discuss. 171, 81 (2014). 